\title{
Intelligent user identity authentication in vehicle security system based on wireless signals
}

\author{
Jie Zhang ${ }^{1,2,3}\left(\mathbb{D} \cdot\right.$ Zhongmin Wang $^{1,2,3}(\mathbb{D}) \cdot$ QingLi $\operatorname{Yan}^{1,2,3}$ (D)
}

Received: 13 September 2021 / Accepted: 11 November 2021 / Published online: 3 December 2021

(c) The Author(s) 2021

\begin{abstract}
Intelligent identity authentication in vehicle security systems, as a vital component in anti-theft system and safety driving assist system, has received wide attention. Current vehicle security systems, however, focus the car security on the car keys security, ignore the owner of car keys. Anyone who owns the car keys can operate the car. This paper introduces an intelligent identity authentication method for vehicle security system based on wireless signals. Unlike past work, our approach combines car security with car owners and car keys. The intuition underlying our design is that when a user walks towards the car, the user's gait information can be leveraged to identify the user. We capture the user's gait information using wireless devices which can be deployed in the car, and then extract features from the received wireless signals using convolution kernel and apply artificial neural network to identify the user. We built a prototype and experimental results show that our approach can achieve high accuracy and strong robustness.
\end{abstract}

Keywords Intelligent system · Identity authentication · Vehicle security · Wireless signals · Machine learning

\section{Introduction}

Vehicle anti-theft is always a major problem in car industries, and many industries are looking to address this problem and improve the security of the car. To allow a vehicle system to distinguish between legitimate users and others, most car companies choose to give passwords to authorized users. For example, Texas Instruments Incorporated (TII) in America used radio frequency technology to develop "vehicle fixed system", and Mercedes-Benz company in Germany used infrared remote controller as the electronic car

Jie Zhang

jiezhang@xupt.edu.cn

Zhongmin Wang

zmwang@xupt.edu.cn

QingLi Yan

yq1@xupt.edu.cn

1 School of Computer Science and Technology, Xi' an University of Posts and Telecommunications, Xi' an 710121, Shaanxi, China

2 Shaanxi Key Laboratory of Network Data Analysis and Intelligent Processing, Xi' an 710121, Shaanxi, China

3 Xi' an Key Laboratory of Big Data and Intelligent Computing, Xi' an 710121, Shaanxi, China key. Unfortunately, car thieves have found some ways to defeat sophisticated locks and alarms. For example, some car thieves use jammers to jam key signals when the car owner locks the car, making it impossible to normally close the door lock and allowing the thieves to take advantage of it. Besides, car thieves can get the car keys from drivers through either force or threat and then resort to stealing cars, which will lead to severe economic losses.

Intelligent identity authentication for vehicle security systems has recently received much attention and many applications would benefit from the identity authentication system. For example, when the car detects opening the car with the car keys remotely, then it will further distinguish whether the key owner is the legitimate user or the others. Thus, even when the car thieves have the car keys, they will not successfully operate the car. Besides, when a smart car detects which legitimate user operates the car, it will trigger some person-specialized customization applications, such as adjust the car temperature and provide content recommendation on car music.

This paper introduces CarAu, an intelligent identity authentication system that works for authentication (distinguish the car key owner is the legitimate user or others) and identification (identify the owner out of the $n$ known users), and the system can be embedded in current vehicle systems. 
Unlike past vehicle security systems, which consider the car owner is the owner of the car keys and proposed a great number of technologies to improve the security of car keys, $\mathrm{CarAu}$ uses both car keys and user identity to confirm the identity of the key owner.

CarAu leverages wireless signals to identify the key owner, as shown in Fig. 1. When the car detects the car keys opening cars remotely, $\mathrm{CarAu}$ system will be run to collect user's gait information while the user walks towards the car. There are two key approaches in wireless signals based human identification, received signal strength, i.e., $R S S$, and channel state information, i.e., CSI. In this paper, we choose CSI-based human identification method because of the finegrained information that $C S I$ can be obtained compared to $R S S$ and higher precision results $[1,2]$.

In line with other CSI-based human identification systems, CarAu employs a transmitter and a receiver that are separately used to transmit and receive wireless signals. The transmitter and receiver are commercial-off-shelf devices and can be equipped in the central control desk of the car. Figure 1 shows the signal propagation path in CarAu. As shown in the figure, wireless signals are transmitted to the receiver via multiple paths, which is called multipath effect. Different user has different gait information, and when different user walks towards the car, the wireless signals arrive receiver through different multiple paths. Different multipath may cause different constructive or destructive interference, which will lead to different multipath profiles, also called CSI measurements. Multipath profiles can be leveraged to identify the user.

After receiving CSI measurements, how can we extract the features and then identify the user? To do so, adjacent subcarrier difference is calculated and a convolution kernel is used to align and extract the features, and then artificial neural network $(A N N)$ is leveraged to identify the user. The $A N N$-based identification model is first trained offline, and then used online. The model is trained separately using the samples from legitimate users and illegitimate users, and car user members.

Summary of results We built a prototype of CarAu using commercial-off-shelf devices as transmitter and receiver and evaluated it with a deployment of two commercial devices in Volkswagen lamando car. Our experimental results show that our approach works well in different scenarios and can achieve a high identification accuracy.

Contributions This paper makes the following contributions.

- It presents an intelligent identity authentication method for vehicle security system based on wireless signals. As a result, the design delivers high identification precision.
- It demonstrates that $A N N$ can work well for human authentication and identification systems, especially when there exist obstacles between users and wireless devices.

- The extract features, i.e., adjacent subcarrier difference of CSI measurements, are robust across walking paths and environmental changes.

\section{Motivation}

Without relying on use of cameras or other additional dedicated devices and by only utilizing non-vision commercial devices, $C a r A u$ can be able to identify the key owners, such as distinguish whether the key owner is the legitimate user or others, or identify who will operate the car. How could such functionality of $\mathrm{CarAu}$ assist the vehicle system in terms of convenience and safety and without using cameras and other dedicated devices at all? Given below are two proof-ofconcept applications of $\mathrm{CarAu}$ that enhance the convenience and safety of auxiliary authentication vehicle system.

\section{Key owner authentication}

Key owner authentication-identify the key owner is the legitimate user or the illegitimate user (the car thief or others)-is one of the main reasons for intelligent identity authentication in vehicle security system. A report from National Insurance Crime Bureau shows that as the vehicle becomes an essential part of our daily life, vehicle theft rate is very high, and nearly 1.3 million cars were stolen in the USA in 2003 and the vehicle theft is also increasing day by day [3]. In this application, $\mathrm{CarAu}$ provides the functionality for distinguishing the identity of the key owner before the key owner opens the car door. If the vehicle system detects an illegitimate user, the system will send a message to the car owner's phone to give an alarm.

\section{Car user identification}

In this application, we investigate the car user identification problem using wireless signals. Specifically, we address the following question - can we identify the user out of the $n$ known users simply using wireless signals? We are interested in the scenario of smart cars where known several users share the car, such as generally the car is shared by $2-5$ car members. $C a r A u$ enables many potential applications that require user identification. For example, a smart car can recognize the car user using $\mathrm{CarAu}$, based on the user identity, it can automatically adjust the background music and temperature, and can also give other personal-specialized recommendations. 


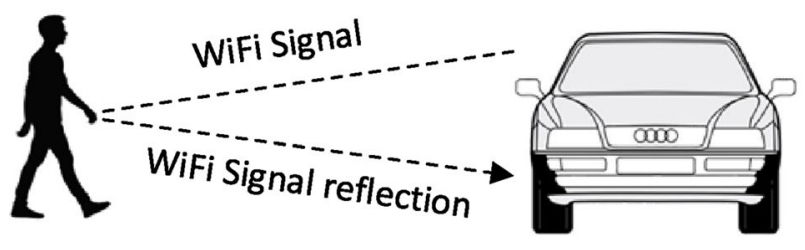

(a)

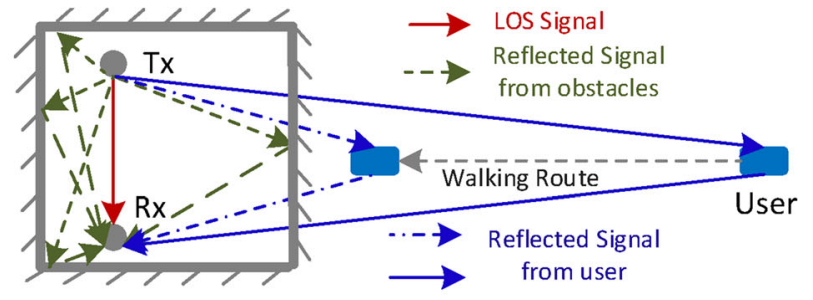

(b)

Fig. 1 CarAu rationale. When one moves towards car (a), multipath effect will lead to different distortions (b), which can be used to identify users

\section{Preliminary}

\section{Channel state information}

CSI describes how the wireless signals propagate from wireless transmitter to receiver, and how the surrounding physical environment affect the wireless signals [4]. In a system with a transmitter with $N_{T X}$ transmit antennas and a receiver with $N_{R X}$ receive antennas, the system will receive $N_{T X} \times N_{R X}$ $\times N_{S}$ subcarriers at the same time in the receiver end, while $N_{S}$ be the number of the subcarriers in each $T X-R X$ channel.

Generally, the system uses wireless NICs (Network Interface Cards) to continuously monitor the variations of wireless signals [5]. Let $X(f, t), Y(f, t)$ and $H(f, t)$ separately be the transmit signal, the received signal, and the measured channel frequency response in different frequency $f$ at time $t$, we can get the following equation [6]:

$H(f, t)=\frac{Y(f, t)}{X(f, t)}$

Since CSI estimates the channel properties of a communication link in wireless communication, and it captures the multipath signals scattered from the obstacles and other surrounding objects, the user activities will generate a unique pattern in time-series CSI measurements, which can be leveraged for human authentication and identification.

\section{How user activities affect wireless signals}

CSI-based human authentication and identification methods rely on repeatable activities and consequently similar CSI measurements. However, in vehicle system, the wireless devices need to be deployed in the car, and the system need to identify the user before the user operates the car. That means the user and the wireless devices will be not in LOS scenario and there will exist obstacles between wireless devices and the user. However, it is not clear that how user activities will affect the wireless signals and how CSI measurements will reflect their characterizations in NLOS scenarios where there exist obstacles between wireless devices and users. In $\mathrm{CarAu}$ system, the obstacles are the car body which is made by metal materials, and it has negative effects on the signal propagation. Through above analysis, in the following section, we will deeply seek the connections between user activities and the received CSI measurements in CarA $u$ system.

\section{User activities}

To distinguish the key owner is the legitimate user or the other, the system needs to make a decision before the key owner opens the car door and operates the car. Before that, the owner needs to walk towards the car, besides, prior researches have also demonstrated that the system can successfully identify the person using the user gait information, such as walking speed, gait cycle time, footstep length and other gait information $[7,8]$ when using wireless signals. Thus, in CarAu system, the human gait information can be leveraged to authenticate the user.

After $C a r A u$ system makes a decision, the owner will open the car door and then operate the car, and to recognize who will operate the car to give some recommendations, the system can also use user gait information to identify the user.

\section{Investigation of CSI}

To link the CSI measurements to user activities, we conduct an experiment to study the relationship. We deploy two wireless devices, one is smart phone with one antenna as the transmitter, and another is the a small computer equipped with Intel 5300 NIC and three antennas as the receiver. The transmitter sends the packet every $2 \mathrm{~ms}$ to the receiver and the receiver can get 500 packets per second. The transmitter and the receiver are in the same position and are all equipped in the central control desk of the car. The system can capture 90 subcarriers in all at each time stamp. We will introduce the observations and discussions in the following:

User authentication and identification User activities are dynamic activities, and they can easily be captured by obvious fluctuations in wireless signals when the user in LOS scenario or near the LOS path. However, in vehicle systems, 
there exist metal obstacles between the user and the wireless devices, and when the key owner walks towards the car before opening the car door, the gait information cannot be described by the obvious fluctuations in wireless signals. We investigate all the subcarrier information of human gait $C S I$ measurements for different persons in $\mathrm{CarAu}$ system, and the results are shown in Fig. 2, the subcarrier information from different users are obviously different, which is the key to authenticate and identify the user. The human gait is reflected by the CSI measurements of all the subcarriers, which are used as fingerprint for human identity.

The impact of the user's walking path In current CSI-based gait authentication and identification systems, the users need to walk on a predefined path and walk in a predefined direction, and the classification models must be trained and tested in the same walk path and walk direction. However, in vehicle system, the user may walk towards the car from multiple directions. The amplitude difference between subcarriers may be caused by human motions and inspired by multipath effect and inspired by prior $C S I$-based localization system [9], we calculate the amplitude difference between adjacent subcarriers and the results are shown in Fig. 2, the subcarrier difference among different paths tends to be the same. Thus, subcarrier difference can help to authenticate the user when the user walks towards the car in different paths, and the model can be trained and tested in combined dataset that consists of multiple path measurements.

The impact of surrounding environment The next challenging problem is that although the wireless devices are deployed in the car and the car layout will not change too much, the vehicle parking environment will always change. Sometimes, there is a car parking in the adjacent parking space and sometimes there is empty. From the analysis above, we know that amplitude difference may also be caused by specific environment, we investigate the adjacent subcarrier difference and the results are shown in Fig. 2, the subcarrier differences among different surrounding environment are similar, which can be used to authenticate the user no matter how the surrounding environment changes. When the user walks different paths and when vehicle parking environment changes, the collected CSI measurements will also be affected, and we evaluate the robustness of the adjacent subcarrier different in one month and the results show that the accuracy does not change too much.

The impact of wireless devices deployment The wireless devices are deployed in the car and can be embedded in current vehicle systems. The transmitter and the receiver can be deployed in the same position and can be embedded in the central control desk or other positions, besides, the transmitter and the receiver can also be deployed in two separate positions. The deployed positions of the wireless devices also affect the received CSI measurements. The results of adja- cent subcarrier difference are shown in Fig. 2, the subcarrier differences among different deployments are obviously different, and the classification models trained for the given deployment cannot be used to test the samples for another given deployment because of the changes of the fingerprints for the two given deployments. However, the wireless devices can be embedded in vehicle systems, and once deployed, the positions will not be changed, and the investigation provides an insight how to find a proper deployment for wireless devices. Figure 2 also shows that compared to deployment 2 (the wireless devices are deployed in two separate positions), subcarrier difference in deployment 1 (in the same position and in central control desk) will be easier to authenticate the user because of more obvious fluctuations in CSI subcarrier difference.

\section{System design}

\section{The framework of CarAu}

Figure 3 shows the whole framework of $\mathrm{CarAu}$, which consists of three main phases including data collection and noise removal, activity detection and feature extraction, human authentication and identification. The basic strategy of $\mathrm{CarAu}$ is hitting two birds with one stone. On the one hand, it analyzes the wireless signals to distinguish whether the key owner is the legitimate user or the illegitimate user. On the other hand, if the key owner is the legitimate user, then it will identify the key owner out of known car users and provide personal-specialized recommendations. First, our system constantly monitors the sensitive events (open the car remotely using the car key), and when the sensitive events are detected, the system will startup automatically to collect the data. To prevent theft thieves, who may not have the car keys, the system can also be implemented as a background service for current vehicle operation systems and the system is always in working state. Then, the system will remove the noise after collecting the CSI measurements, and then fingerprint features will be extracted and used to identify the user using a convolution kernel and $A N N$. We will introduce the details of the system design in the following section.

\section{Data collection and noise removal}

CarAu system is always running in the background and when detects sensitive event, $C S I$ measurements are collected. The system collects CSI measurements on the receiver end of a wireless link using two wireless devices, which one is as the transmitter and another is as the receiver. Current CSIbased authentication and identification systems collect CSI measurements using Linux 802.11n CSI tool, which uses Intel 5300 NIC $[10,11]$, or Atheros CSI tool, which uses 


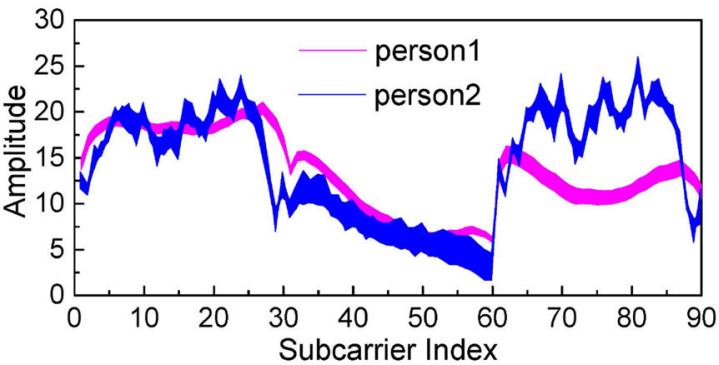

(a)

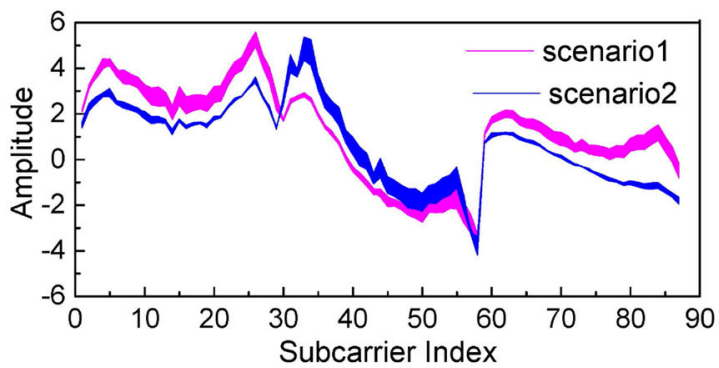

(c)

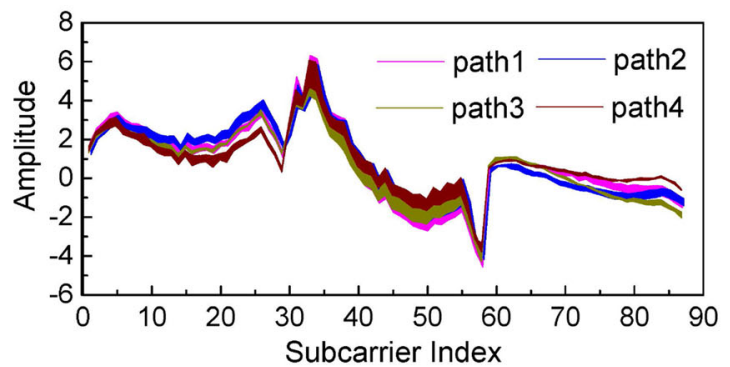

(b)

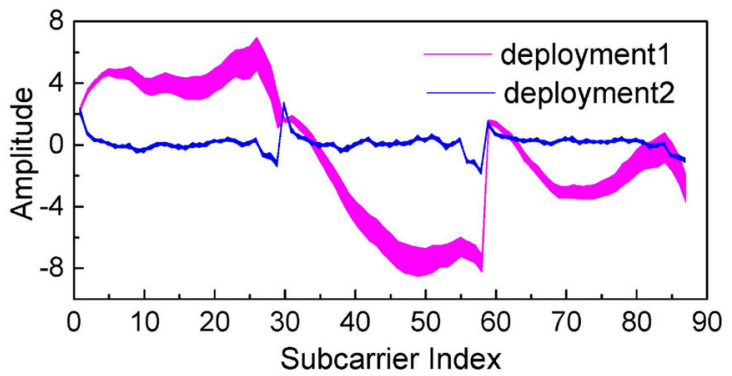

(d)

Fig. 2 Subcarrier difference. a Subcarrier information among different persons. b Subcarrier difference among different walking paths. c Subcarrier difference among different surrounding environment. d Subcarrier difference among different deployments

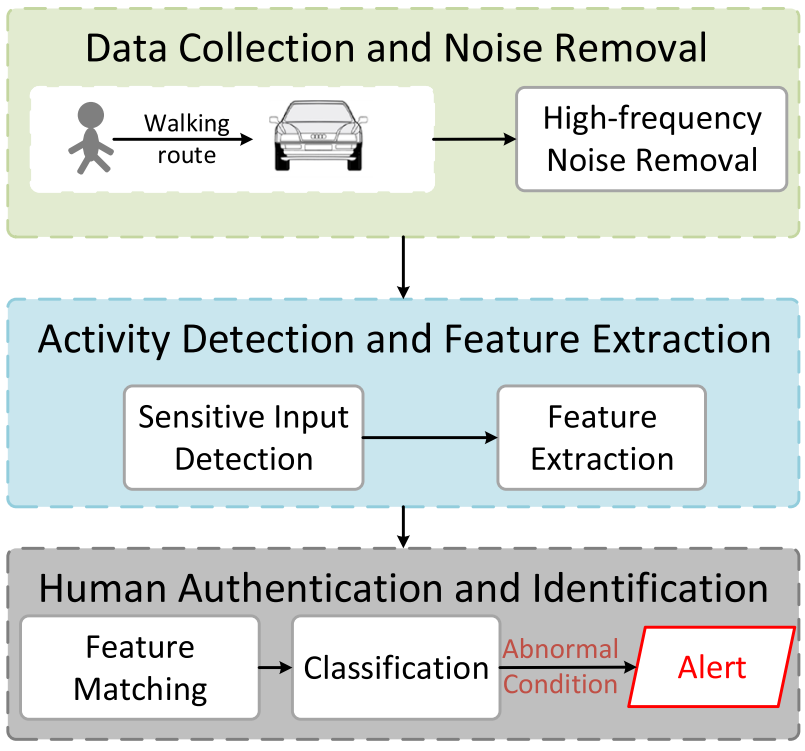

Fig. 3 The architecture of the proposed identify authentication framework $\mathrm{CarAu}$

Atheros NICs [12,13]. Generally, the receiver is a laptop or PC, which equipped with NICs. For CarAu system, we use Intel 5300 NIC with three antennas as the receiver and a smartphone with one antenna as the transmitter. For each pair of transmit antenna and receive antenna, there are 30 subcarriers and we will have $1 \times 3 \times 30=90$ subcarriers in total. The CSI time-series sequence for each subcarrier of a given pair of transmit antenna and receive antenna is called a CSI stream. As the system sends a packet per $2 \mathrm{~ms}$, thus, the system receives 500 packets for 90 CSI streams per second.

Because of the frequent changes in transmit power levels, internal CSI reference levels, transmission rates, etc., the collected CSI measurements often have the inherent noise in time and frequency domain. Thus, the collected CSI measurements need to remove the noise for the further feature extraction and authentication and identification. In this paper, discrete wavelet transform is used to remove the noise $[14,15]$ and the details are introduced in the following.

Generally, the steps of noise removal using discrete wavelet transform include forward wavelet transform, threshold quantification and inverse wavelet transform. First, the original signal is divided into approximation coefficients and detail coefficients, which is an iterative step. Then, process the coefficients using threshold and finally reconstruct the signal using coefficients. Given the distortion of signals, the effectiveness and simplicity of soft threshold, the good characteristics of Symlets wavelet filter, Symlets wavelet filter of order 10 is applied to remove the noise in this paper and soft threshold is used in threshold quantification phase.

\section{Convolution kernel-based feature extraction}

Existing researches use statistics feature [8,16,17] (e.g., mean, maximum, and deviation), or more sophisticated feature, such as DWT feature $[6,18]$ and frequency feature 
[7] to describe the characteristics of wireless signals, and unfortunately those features cannot fulfill the requirements in vehicle systems. Inspired by Lasagna system [19], which leverages mobile sensor data to recognize human activity, and Deepsense system [20], which solves the noise removal and feature customization problems while using mobile sensor data, we use a convolution kernel to customize the features for user activities.

When a user performs activities, the receiver continuously collects CSI measurements. Since CarAu system relies on the collected fingerprints, which are set as the training set in authentication and identification phase. There will be no various variations on CSI measurements while the target walks towards car compared to other CSI applications in LOS scenes. We found that compared to subcarrier amplitude, adjacent subcarrier difference can be used to detect the target's walking, inspired by prior work [21,22], we calculated subcarrier difference of adjacent subcarriers and it can be calculated according to the following equation:

$$
\begin{aligned}
& \Delta s d_{j}=a m_{i+1}-a m_{i} \\
& 1<i<89,1<j<89
\end{aligned}
$$

where $s d_{j}$ is the adjacent subcarrier difference of subcarrier $i+1$ and subcarrier $i, a m_{i+1}$ is the amplitude of subcarrier $i+1$, and $a m_{i}$ is the amplitude of subcarrier $i$.

In authentication and authentication model, the user need to perform the activities multiple times to collect training data, and the CSI measurements collected each time may be in an unsynchronized way, which is caused by the different performing time each time. Before putting these data into authentication and identification model, we need to pack the adjacent subcarrier difference of CSI measurements into a coordinated structure.

The CSI measurements and adjacent subcarrier difference of CSI measurements can be represented in the following format:

$H=\left[h_{1}, h_{2}, \ldots, h_{i}, \ldots, h_{90}\right]$

where $h_{i}$ is a $C S I$ stream.

$S D=\left[\Delta s d_{1}, \Delta s d_{2}, \ldots, \Delta s d_{j}, \ldots, \Delta s d_{89}\right]$

where $\Delta s d_{j}$ is the difference of two adjacent subcarriers.

Data from the same activities that collected multiple times are organized in a data matrix $D$, which can be represented in the following format:

$D=\left[S D_{1}, S D_{2}, \ldots, S D_{i}, \ldots, S D_{m}\right]$ where each $S D$ can be seen as the difference matrix of a CSI measurement for the activity, and $m$ is the collected times of CSI measurements of the activity.

Assume that the performing time of the activity is $t$ and the activities are performed $m$ times, the length of received $C S I$ measurements, i.e., a $C S I$ stream $h_{i}$, will be $500 t$. The size of matrix $H$ will be $500 t \times 90$, matrix $S D$ is $500 t \times 89$.

As shown in Fig. 4, first amplitude difference between adjacent subcarriers is calculated and then we pack the adjacent subcarrier difference of CSI measurements for the same activities that are performed multiple times for the same user to data matrix $D$. The size of matrix $D$ is $500 t \times 89 \times m$. Then, the matrix is convoluted with a group of convolution kernels to align and extract the features. The convolution kernel can capture the local and consecutive features of subcarrier differences by convolving $S D$ with a set of kernels of different sizes.

The group of convolution kernels $R$ can be obtained through the following equation:

$f(x)=\frac{1}{\sigma \sqrt{2 \pi}} e^{-\frac{(x-\mu)^{2}}{2 \sigma^{2}}}$

The group of convolution kernels obey orthodox distribution and the range is from 0 to 1 . For each series of data of input data matrix $D$, the kernels are applied to perform convolution operation to align the data structure and extract features [19]. Convolution kernels $z \in R^{w}$ is applied to a window of $w$ packets to generate a local feature value. The convolution operation can be calculated as the following equation:

$m_{i}=\operatorname{Cov}\left(z^{T} d_{i:(i+w-1)}\right)$

where $d_{i:(i+w-1)}$ denotes concatenated vectors $\left[d_{i}, d_{i+1}, \ldots\right.$, $\left.d_{i+w-1}\right]$ of matrix $D$, and $m_{i}$ is the computed feature value at position $i$. A feature map $F \in R^{n-w+1}$ can be obtained by computing $m_{i}$ at all possible positions, and feature map $F$ can be represented as $F=\left[m_{1}, m_{2}, \ldots, m_{n-w+1}\right]$.

\section{ANN-based authentication and identification}

CarAu system uses an artificial neural network (ANN) [23] to do user authentication and identification. The inputs of the classification model are the extracted features and the outputs are the labels that are legitimate users or illegitimate users in human authentication, car user members in user identification. Generally, building and using a classification model consists of two steps, train the model and then use the model, no matter what kind of the classification model is, such as traditional machine-learning algorithms or $A N N$-based learning algorithms. 
Fig. 4 The framework of feature extraction

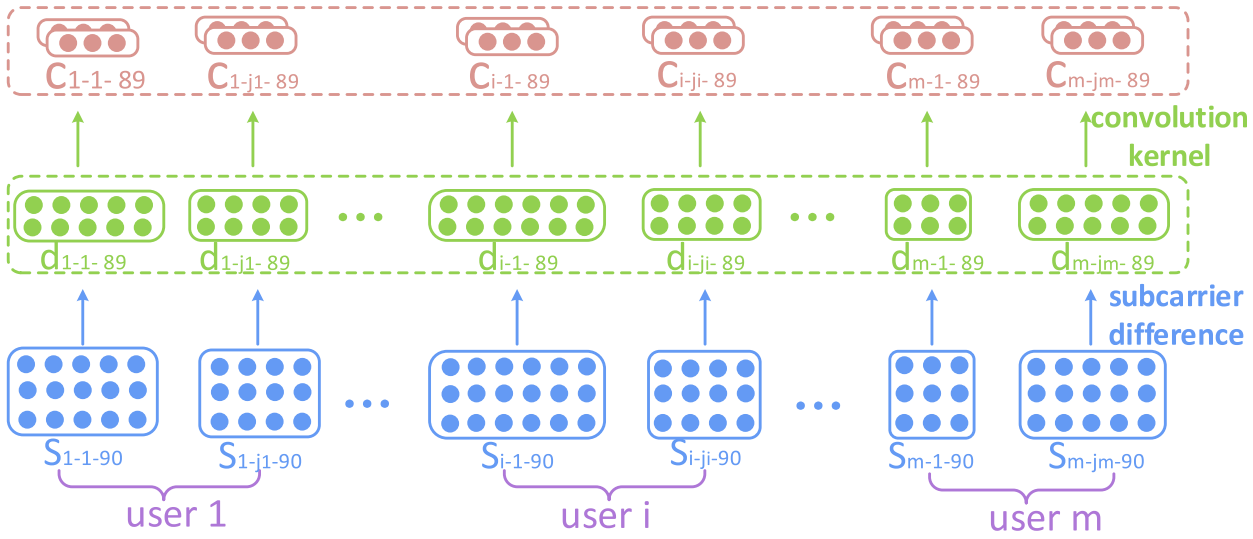

Figure 5 shows the structure of user authentication model and identification model, which is a fully connected, feedforward ANN. As shown in Fig. 5, ANN is composed of input layer, hidden layer and output layer. The input of $A N N$ is extracted features, and the output is predicted results. Assume that input vector can be represented as $D_{s}=\left[d_{1}, d_{2}, \ldots d_{l}\right]$, output vector can be represented as $\hat{u}=\left[u_{1}, u_{2}, \ldots, u_{n}\right]$, the output of $A N N$ can be obtained according to the following equation:

$\hat{u_{j}}=f\left(\sum_{i=1}^{n} \omega_{i j} x_{i}-\theta_{j}\right)$

where $\omega_{i j}$ is weights between neuron $i$ and output neuron $j$, $x_{i}$ is output is neuron $i, \theta_{j}$ is the threshold of output neuron $j$, and $f$ is activation function of output neuron.

In $C a r A u$ system, the number of nodes of input layers is determined by the length of the extracted features while the number of the nodes of the output layers is 2 in human authentication model and in user identification model, the number of the nodes of the output layers is determined by the user set.

The goal of ANN training is to obtain a set of appropriate weight values to minimize the error between output results and true values, and take mean square error between the output value and the true value as the loss function, the loss function can be obtained according to the following equation:

$E=\frac{1}{2} \sum_{j=1}^{n}\left(\hat{u_{j}}-u_{j}\right)^{2}$

where $\hat{u}_{j}$ and $u_{j}$ are, respectively, predicted value and true value.

To minimize the loss function, the weight value and offset value need to be constantly adjusted to make the actual output closer to the expected output to reduce the loss error. The authentication and identification model are trained using back-propagation with Stochastic Gradient Descent (SGD). The network parameters which can minimize the error of loss function are found through multiple iterations, and the network parameters $\Theta$ can be obtained according to the following equation:

$\left.\Theta=\underset{\Theta}{\arg \min } \frac{1}{n} \sum_{i=1}^{n} \ell\left(X_{i}, \Theta\right)\right]$

where $X_{i}(0 \leq i \leq n)$ is a set of training examples, $\Theta$ is the output values of minimization loss function found by continuous iteration, and $\ell\left(X_{i}, \Theta\right)$ is the loss function.

The neural network minimizes the error of loss function through continuous iterative training. When minimum error of loss function is obtained, the error of each neuron and the whole network also reaches the minimum, the network training function also reaches the best training result, and learns an optimal mapping relationship between the input and the output, the mapping relationship is the trained model what we need for authentication and identification model.

The training data of the model are collected from the legitimate users and some illegitimate users in human authentication model while they are collected from the car users (e.g., family members) in user authentication model. The human authentication model learns from the training data how to map a CSI measurements to legitimate user and illegitimate user while the user identification model learns how to map a CSI measurements to $n$ known car users. Once the model learns the mapping, the model can be easily be used to the testing CSI measurements.

Cost of time and space The training cost of the two models come from two parts: gathering and preprocess training measurements, and building classification models. Gathering and preprocess the training measurements can run offline and building the classification model take less than 5 min on a multi-core server. The model can be built offline and then predict the testing CSI measurements online. The trained model can be saved in vehicle systems, and it spends less than 3 


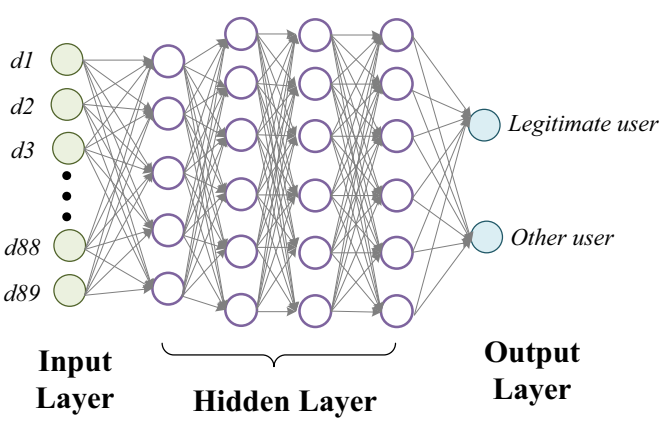

(a)

Fig. $5 A N N$-based user authentication model and identification model

$\mathrm{MB}$, and authenticate and identify the user using the trained model just take less than $1 \mathrm{~s}$ on a multi-core server, which is acceptable for authentication and identification system.

\section{Experimental setup and evaluation}

In this section, we present the experimental setup and the evaluation metrics used for the evaluation. For evaluation part, we first conclude the overall performance and then consider the impact of alternating quantity on system performance, such as feature extraction method, recognition method and other environmental factors.

\section{Experimental setup}

Scenarios We evaluate our system in various outdoor car parking scenarios, described as follows. The car is parked in various positions in the parking scenarios, as shown in Fig. 6. Besides, when the car is parked in a place, there are several scenarios need to be considered. The first scene is that there is a car parking nearby the user's car while the second scene is that there is no car parking near the user's car. When a car is parked in a place, we conduct the experiment for several days, and the parking environment experiences different changes.

Wireless device setup As an example, Fig. 6 shows the wireless device setup in CarAu system. To collect CSI, we use XiaoMI Note2 as the transmitter and use Intel 5300 NIC equipped with a mini $\mathrm{PC}$ as the receiver. The transmitter is equipped with one transmit antenna and the receiver is equipped with three receive antennas, and the transmitter sends the packets at the rate of 500 packets per second. In $\mathrm{CarAu}$ system, the transmitter and the receiver are deployed in two deployments, and one is that the transmitter and the receiver are deployed in the same position, which can be embedded in central control desk-Pos 1 as shown in Fig. 6.

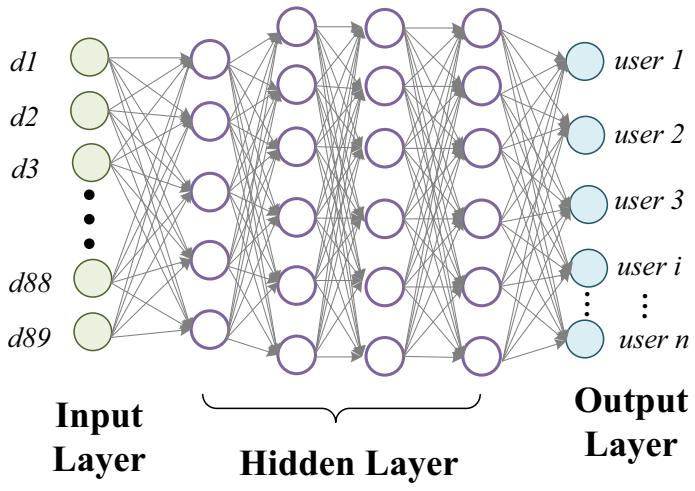

(b)

Another is that the transmitter and the receiver are deployed in two positions, and one is deployed in central control desk, and another is deployed in air conditioner wind outlets, which is $\operatorname{Pos} 2$ in the figure.

Participants We recruited 20 volunteers (12 males and 8 females) to participate our experiments, which are with the approval of REB (research ethics board). Each participant was given the opportunity to practice the activities several times, so that they could perform the activities at their natural speed. In the experiment, each participant was asked to walk repeatedly on a straight line and then open the car door and sit in the driver's seat. The participants were asked to walk in their natural speed without intentional speed up or slow down. Besides, the participant walked towards the car from multiple directions, as shown in Fig. 6. Each participant performed each activities 20 times in each walking direction and each device deployment.

Model evaluation In user identification model, we consider different group sizes starting from 2 to 5 users. These group sizes were chosen based on the number of users sharing the car for family members (2-4) or company members (3-5). For each group size, we use cross-validation to evaluate $C a r A u$ system. We compare CarAu against two current feature extraction methods, which are used in state-of-art CSI-based gait identification system, and four machine-learning algorithms, which are frequently used in user identification cases.

Evaluation metrics To scientifically describe the system performance, we also consider True-Positive Rate (TPR) and False-Positive Rate (FPR) two reasonable evaluation criteria. TPR represents the fraction of the cases that $\mathrm{CarAu}$ can correctly authenticate and identify the user while FPR represents the proportion of the cases that $\mathrm{CarAu}$ mistakenly takes measures when the user is legitimate. 


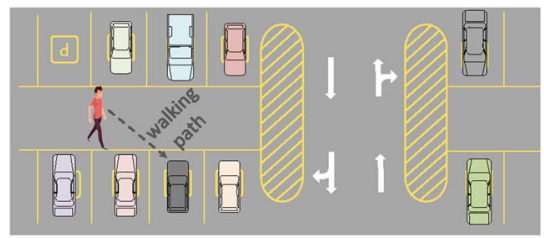

(a)

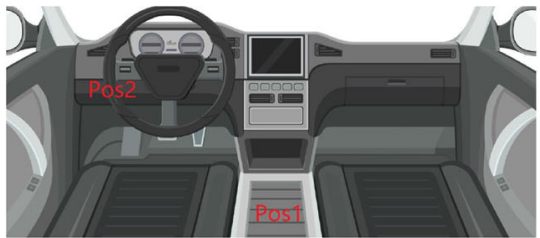

(b)

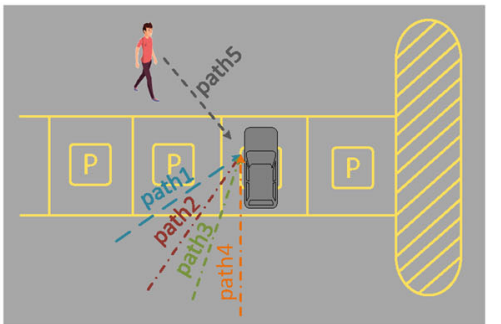

(c)

Fig. 6 Experimental setup. a Car parking scenario. b Device deployment. c The user walks towards the car from multiple directions

\section{Main findings of evaluation}

The main findings of evaluation are as follows:

- CarAu achieves over 96\% accuracy for human authentication. The results show that the system can successfully distinguish legitimate user and illegitimate users, and the system can prevent vehicles from being started and driven away by illegitimate users by adding some protection measures when detected a illegitimate user trying to operate the car, and it improves the safety of vehicle systems to a certain extent.

- CarAu achieves over 92\% accuracy for user identification. The results show that the system can successfully identify who will drive the car. CarAu delivers consistently performance no matter the wireless device deployment, environmental changes and user's walking paths.

- Our system is intelligent compared to other vehicle security systems, The intelligence of the system is reflected in that it does not need the cooperation of users and other professional equipment, which can be carried out without user's knowledge. However, our system only provides a prototype framework, and more intelligent functions need to be developed in the future, such as the system can lock the car when detected illegitimate users trying to operate the car even though he/she has the car key, give some personal-specialized recommendations when detected a legitimate car user.

\section{Overall performance}

In all experiments, we use cross-validation to make sure that the training dataset and testing dataset are different. For human authentication, the legitimate users need to collect training measurements to build the training dataset in training phase while not all illegitimate users have the training measurements in the training dataset, and in the experiments, we test the testing data of illegitimate users using the dataset which does not include the data of tested illegitimate users,
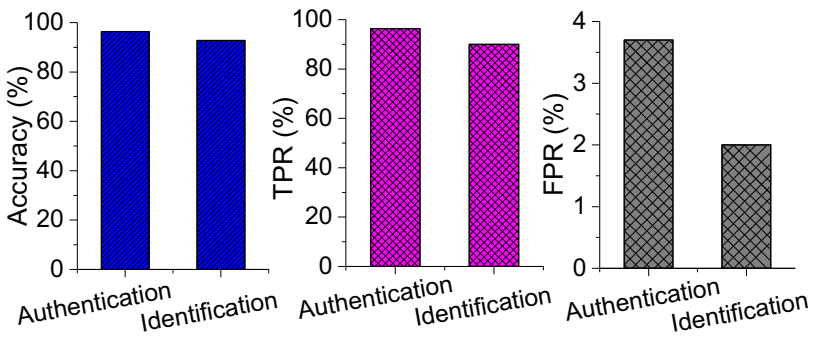

Fig. 7 Overall performance, includes accuracy, TPR, FPR for human authentication and identification

and it is in line with actual cases. For user identification, all the car members need to collect training dataset.

Our results show that CarAu can detect a legitimate user and illegitimate user at an accuracy of over $96.4 \%$ and can identify the user at an accuracy of about $92.8 \%$.

The accuracy of human authentication can achieve over $96 \%$, and the accuracy of user identification can achieve about $92 \%$, seen from Fig. 7. That is because compared to authentication task, where the system just only need to determine whether the testing user is the legitimate user or not, identification task is considerably harder, where the system has to recognize which user the testing user is. Besides, we try to add the training data of the tested illegitimate user into training dataset, and we have the finding that after adding the training dataset, the accuracy of human authentication can achieve about $98 \%$. The results show that if we can continuously add the data of legitimate user and illegitimate user into training dataset, the system can achieve a higher human authentication accuracy. That can be implemented in an internet-of-vehicle systems, and the system can report the data of the illegitimate users to all the vehicles.

Our results show that CarAu achieves average TPR and FPR of about $96 \%$ and $3.7 \%$ for human identification, $90 \%$ and $2 \%$ for user identification, respectively.

Figure 7 also shows the distribution of TPR and FPR for human authentication and user identification. CarAu successfully gives the car owner a warning of 96\% with TPR 96\% and gives the car user proper recommendation of $92.8 \%$ with TPR 90\%. 


\section{Feature extraction analysis}

We compare our feature extraction method from the two following aspects: (1) comparison between two current alternative feature extraction methods; (2) the convolution kernel used.

\section{Alternative feature extraction methods}

Our results show that our approach can achieve higher accuracy comparable with current feature extraction methods.

To demonstrate the effectiveness, and evaluate the performance of feature extraction method of CarAu method, we compare our feature extraction method against two current feature extraction methods used in WiFiU [7] and WiWho [8], which both leverage users' gait information to do recognition. Note that in the experiment, the performance of user identify is evaluated when the user group size is 5 , and the testing data is tested using $A N N$.

Figure 8 shows the comparison results of user identification. Using current feature extraction methods, the system fails to recognize users in most cases, and achieves only $64 \%$ and $37 \%$ accuracy. That is because the features calculated using current feature extraction methods are based on CSI amplitude. However, in CarAu system, the received CSI amplitude will not cause large fluctuations, due to the metal car body between the testing user and wireless devices. As a result, the calculated features cannot successfully differentiate users and will lead to low accuracy.

\section{Impact of the size of kernel}

Our results show 500 is a good alternative for feature length when using a convolution kernel to align CSI measurements.

As the size of the kernel will cause impact on the final features, which is the input of classification models, we evaluate the performance of $\mathrm{CarAu}$ with different kernel size. Note that in the experiment, the network structure used in the classification model is the same. Figure 8 shows how the kernel size affects the system performance. As can be seen from Fig. 8, when the feature length is 500, the system can achieve comparable best accuracy, and when changing the kernel size, the system accuracy does not have large improvement or large decrease, and the accuracy keeps stable. In this work, we choose 500 as feature length, but other feature length also works.

\section{Classification model analysis}

We also compare our $A N N$-based classification techniques against current machine-learning techniques and neural network structures.

\section{Alternative model techniques}

Our results show that our approach has an obvious accuracy improvement compared to current classification models.

To evaluate the performance of classification models implemented in $C a r A u$, we compares our $A N N$-based classification techniques against four current frequently used machine-learning techniques, KNN (K-Nearest Neighbor), SVM (Supported Vector Machine), RF (Random Forest), and NB (Naive Bayes). Note that in the experiment, the testing data used the same feature extraction method to calculate the features.

Figure 9 shows the results, different from current classification methods which exhibits low accuracy in human authentication and user identification, $A N N$ achieves higher accuracy. That is because $A N N$ can capture both linear and non-linear relationships between the subcarriers of CSI measurements while current classification models can only capture one of the relationship, which will not be suited to do authentication and identification in vehicle systems.

\section{Impact of neural layers}

Our results show an ANN with 5 hidden layer will be the choice of the system.

As more network layers will help the network to better capture the relationship, but will lead to over-fit. To evaluate the effect of network layers on system performance, we increase the number of layers from 1 to 10 . Note that in the experiment, the convolution kernel structure used to extract features is the same and Fig. 9 shows the results. We observe that using 5 hidden layers can give the best performance. Although there is an improvement in accuracy using different network layers, the improvement is small, and just about $1 \%$ to $2 \%$ improvement. Thus, in this work, we choose to use an $A N N$ with 5 hidden layers, but other $A N N$ structures also work.

\section{Evaluation on robustness}

Our results show that the changes of device deployment, different user walking paths, surrounding environmental changes does not significantly affect the accuracy ofCarAu.

\section{Impact of device deployment}

Our results show the two deployments can both achieve over 90\% accuracy.

In this experiment, the model is trained and tested separately for two different deployment. For the first deployment (the wireless devices are deployed in the same position), the accuracy can achieve about $95 \%$ while $90 \%$ for the second deployment (the wireless devices are deployed in two dif- 
Fig. 8 Feature extraction analysis. a Accuracy comparison between convolution kernel-based feature extraction method and other two different feature extraction methods. b Accuracy comparison between different feature length when using different convolutional kernels

Fig. 9 Classification model analysis. a Accuracy comparison between $A N N$ and other four classification learning algorithms. b Accuracy comparison between different $A N N$ structures
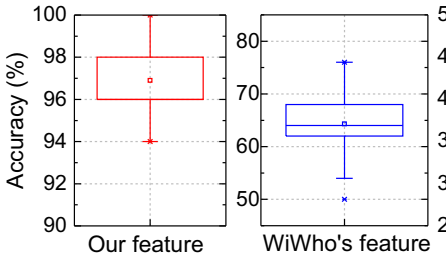

(a)

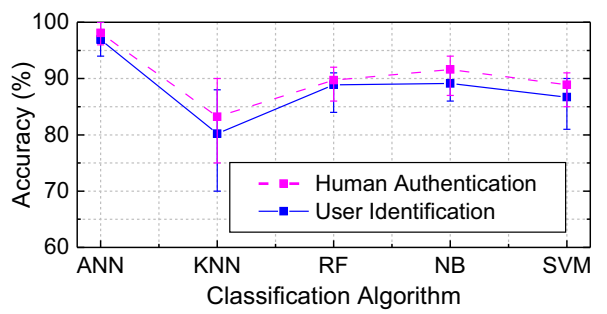

(a)

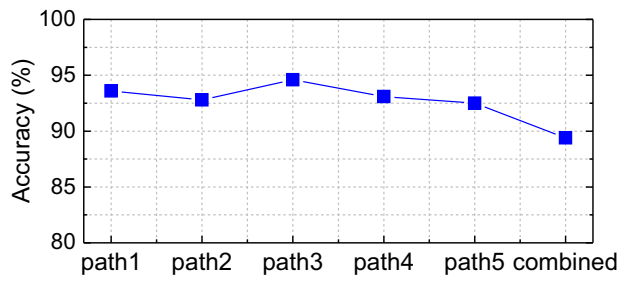

(a)

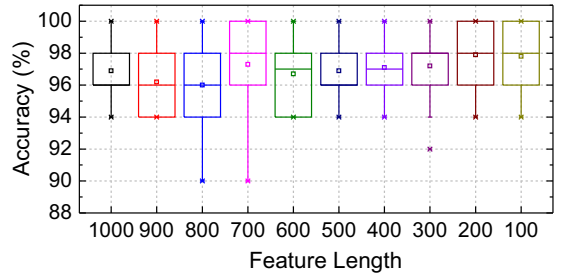

(b)

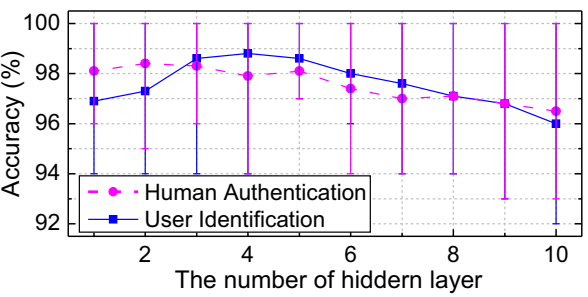

(b)

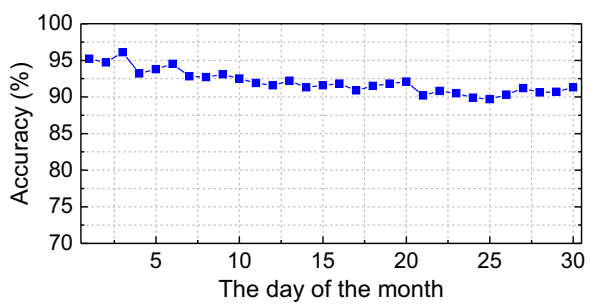

(b) ferent positions). Thus, to achieve higher performance, the wireless devices can be deployed in the first deployment. The results also show the device deployment has little impact on performance.

\section{Impact of different walking paths}

Our results show that the performance of CarAu deteriorates only when the user's walking paths change significantly.

To study the impact of user's walking paths on the system performance, we collect CSI measurements of 5 walking paths, and Fig. 10 shows the results. We found that the accuracy can achieve over $90 \%$ accuracy and will not decrease when user walks different paths, which performs better than current system, such as WifiU. That is because when the user's walking path and directions are similar, e.g., walking path 1 to 4 in Fig. 6, the difference between CSI subcarriers is the same, as shown in Fig. 2. However, when the user's walking path has a relatively big change, e.g., walking path 5 in Fig. 6, the subcarrier difference will also have a great change. Thus, in actual applications, the system can collect the training data of several walking paths, and the user does not have to walk the same path each time, which is impractical in actual cases.

\section{Impact of environmental changes}

Our results show that the changes of surrounding environment do not significantly affect the performance of CarAu.

To evaluate the impact of changes of surrounding environment, we collect the data over months, and the surrounding environment experiences different changes. We first train the classification model using the training measurements of one kind of surrounding environment, and then test the data of another surrounding environment. Figure 10 shows the results, we can observe that the system performance does not decrease and keep stable no matter how the surrounding environment changes.

\section{Related work}

Our work lies at CSI-based human authentication and user identification system, and vehicle security systems. In this paper, we will introduce the related work from the following two aspects. Table 1 shows related work and their differences with CarAu system.

CSI-based authentication and identification systems. User authentication and identification system cannot only prevent 
Table 1 Comparisons between prior work and CarAu System

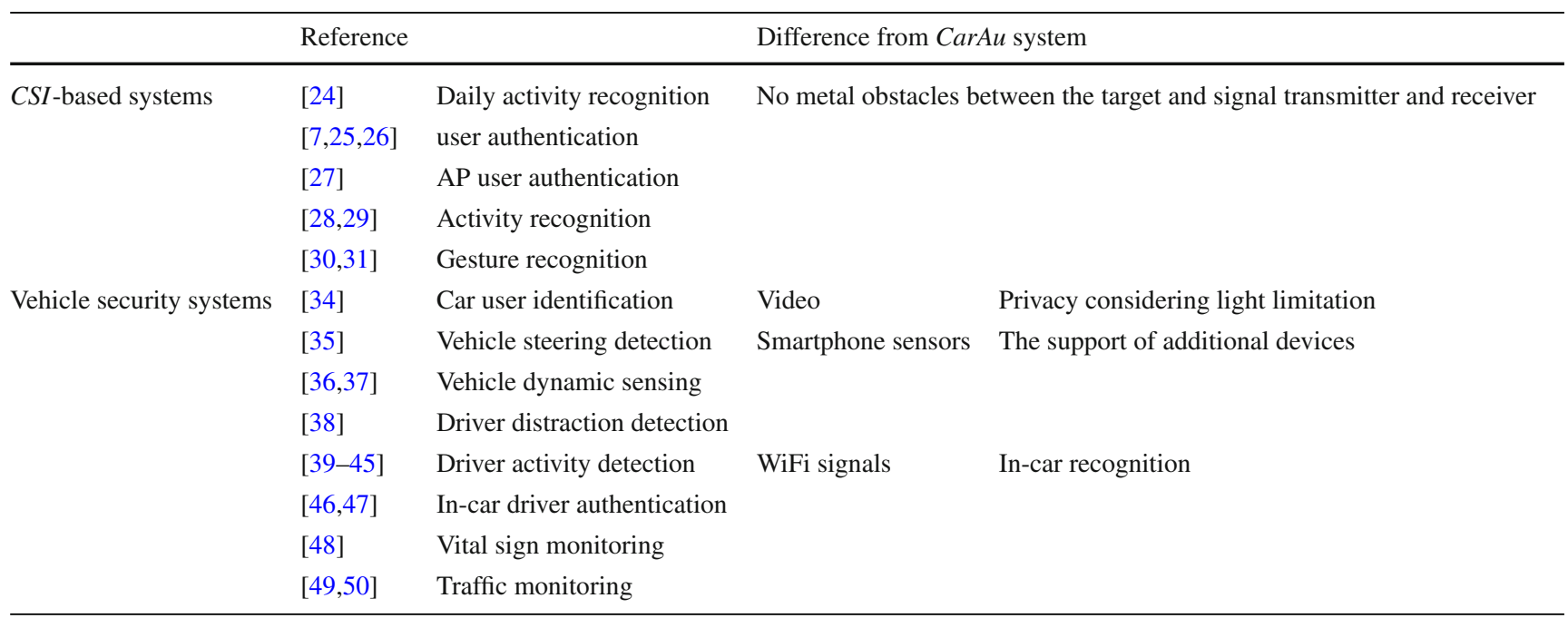

unauthorized users to access confidential information, but also can customize the services for the user. Shi et al. [24] leverage users' daily activities, such as walking and stationary ones to authenticate the user. Liu et al. [25] successfully use $C S I$ to authenticate users in practical environment. WiFiU [7] and WiFi-ID [26] use user's gait information to identify the user. NiFi [27] proposes a non-intrusive user identification system, and the AP can automatically identify legitimate users. Jiang et al. [28] propose an activity recognition system independent of environment while WiAG [29] is agnostic with user's position and orientation. WiFinger [30] and Venkatnarayan et al. [31] recognize the user's gesture separately for one user and multiple users. However, current CSI-based systems recognize the user/activity in LOS and NLOS scenarios where there exists no metal obstacles between the target and transmitter and receiver, which will have stronger impact on wireless signals.

Vehicle security systems. To improve the security of devices in Internet of Things, key, certificate [32] and strategy [33] are proposed. The vehicle security rely on the design to improve the security, which does not distinguish users. Different from those, an image processing system is set in a car to identify car thieves and leverages a device to take clear and full-faced images [34]. However, video-based systems will leak user's privacy and will not work well in dark environment. Besides, V-Sense [35] presents a vehicle steering detection middleware, which uses the commodity smartphones with sensors, and the system can detect how a vehicle is steered and then alarm the drivers in time. Wang et al. [36] and Yan et al. [37] use smartphone to sense vehicle dynamics and then determine driver phone use, which can be used in many traffic safety applications. iLoc [38] presents an infrastructure-free, cooperative and in-vehicle positioning system based on smartphones, which can pre- vent driver distraction, and customize in-vehicle services. However, that will require the support of additional devices, which are expensive. Recently, CSI and smartphones are used in vehicle systems to improve environmental and behavioral awareness, etc. WiBot [39] presents a gesture based personal assistant system for vehicles, which can detect distracted behaviors and uses a gesture to help user interact with the cars. WiFind [40] proposes a fatigue detection approach, and Muneeba et al. [41] detect driver's distracted behavior. WiDriver [42] monitors driver operation on steering wheel using CSI. SafeDrive-Fi [43] predicts driver states through gestures and body movements. WiCAR [44] is a real-time in-car human activity recognition system. CARIN [45] recognizes driver activity under the interference of passengers. Regani et. al $[46,47]$ propose a in-car driver authentication system using WiFi. V2iFi [48] monitors in-vehicle vital sign under driving condition. WiTraffic [49], a traffic monitoring system, can also be designed to classify vehicles, detect lanes and estimate speed. Wang et al. [50] propose a vehicle speed estimation model, which could also monitor the traffic status seamlessly. However, current CSI-based systems focus on the in-car recognition while CarAu system authenticate and identify the user before the helshe opens the car door, which can work well outside the car.

\section{Discussions and limitations}

There is still room for the further work and to enhance system availability and robustness, and we discuss a few points in the following.

Feasibility CarAu system requires fixed deployment of transmitter and receiver. Although in the classification model, the training set can include the fingerprints of different deploy- 
ments, the changes of the device deployment will need to recollect the fingerprints. We believe that will not be an issue since the wireless devices are deployed in the car and can be embedded in current vehicle systems, which will lead to rare changes in the positions of wireless devices. We also assume that the user walks a straight line towards the car. The system runs when the car detects the key opening the car remotely, and the user often opens the car when he/she is close to the car, and a straight walkway can be chosen for the purpose. Changes in user's attire (clothes, shoes, backpack, etc.), weather and other factors associated with the target and data collection environment, may affect the system performance. Signal to Noise Ratio (SNR) can be used to evaluate the system robustness [51], and we have not studied in this paper, which will be our future work.

Surrounding environment CarAu system assumes that there is a single person performing activities, which is the assumption of majority of current wireless signals based sensing systems. When there are multiple users walking at the same time, the CSI measurements received at the receiver end will be the complex value from multiple users and it is difficult to separate the received wireless signals. However, we believe that combined with Venkatnarayan's work [31], Liu's work [52] and Wang's work [53], the problem can be solved, and that is what we need to be solved in the future work to improve the system robustness.

Depending on particular hardware cards CSI measurements need to be collected using particular NICs in Linux operation system, such as Intel 5300 NIC and Atheros NICs. However, wireless transmitter and NICs are commercial devices, and they are very cheap, such as a Intel 5300 NIC just cost about 3 dollars, and the wireless transmitter can be the automotive WiFi with the development of the car vehicles. Moreover, with the deployment of CSI-based sensing systems, the collection can be integrated in one device and can be embedded in current vehicle systems. Besides, with the explode and maturation of CSI-based sensing applications, CSI will be exposed to upper layers using most NICs in the near future.

\section{Conclusion}

This paper present $C a r A u$, an intelligent user identity authentication method for vehicle security systems, which distinguishes the user is the legitimate user or other and then identify the user from known user group to give personalspecialized recommendations. We first show the feasibility and robustness of CarAu system from the perspective of CSI characteristics and then we use convolution kernel to align the CSI measurements and extract features, and use $A N N$ to authenticate and identify the user. We evaluate the performance of $\mathrm{CarAu}$ from effectiveness and robust- ness two perspectives. For effectiveness, we evaluate the model parameters and compare the model used in $\mathrm{CarAu}$ against current used models. For robustness, we evaluate the human authentication accuracy and user identification accuracy from wireless device deployment, different walking paths and environmental changes three perspectives. Experimental results demonstrate the effectiveness and robustness of the system, and it can achieve about $96 \%$ authentication accuracy and $92 \%$ identification accuracy. We believe that combined with multi-users recognition and considering more interference factors in vehicle systems, CarAu system can be more intelligent, and it is out of the scope of this paper and is our future work.

Acknowledgements This work is supported by the Natural Science Foundation of China (62002287), Scientific Research Program Funded by Shaanxi Provincial Education Department (Program No.20JK0912), and Key research and development plan of Shaanxi Province-General Projects (2021NY-036).

Author Contributions Jie Zhang wrote the main paper and conducted the main experiment. Zhongmin Wang modified the paper. Qingli Yan helped to conduct the experiment.

Funding Natural Science Foundation of China (NSFC), 62002281, Jie Zhang Scientific Research Plan Projects of Shaanxi Education Department, 20JK0912, Jie Zhang Shanxi Provincial Key Research and Development Project, 2021NY-036, Qingli Yan Natural Science Foundation of China (NSFC), 61373116, ZhongMin Wang Conflict of interest On behalf of all the authors, the corresponding author states that there is no conflict of interest.

Availability of data and material The datasets used or analyzed during the current study are available from the corresponding author on reasonable request.

\section{Declarations}

Conflict of interest On behalf of all the authors, the corresponding author states that there is no conflict of interest.

Code availability The custom code during the current study are available from the corresponding author on reasonable request.

Ethics approval The experimental protocol was established, according to the ethical guidelines of the Declaration and was approved by REB (research ethics board). Written informed consent was obtained from individual or guardian participants.

Consent to participate The participants gave informed consent to participate in the study.

Consent for publication The data are obtained with the agreement of the participants, and they agreed to publish the study.

Open Access This article is licensed under a Creative Commons Attribution 4.0 International License, which permits use, sharing, adaptation, distribution and reproduction in any medium or format, as long as you give appropriate credit to the original author(s) and the source, provide a link to the Creative Commons licence, and indicate if changes were made. The images or other third party material 
in this article are included in the article's Creative Commons licence, unless indicated otherwise in a credit line to the material. If material is not included in the article's Creative Commons licence and your intended use is not permitted by statutory regulation or exceeds the permitted use, you will need to obtain permission directly from the copyright holder. To view a copy of this licence, visit http://creativecomm ons.org/licenses/by/4.0/.

\section{References}

1. Yang Z, Zhou Z, Liu Y (2013) From RSSI to CSI: indoor localization via channel response. ACM Comput Surv 46(2):1-32

2. He S, Chan S (2017) Wi-fi fingerprint-based indoor positioning: recent advances and comparisons. IEEE Commun Surv Tutor 18(1):466-490

3. USA Today. Top car-theft areas in each state. https://www. usatoday.com/news/nation/2004/11-29-car-thieftable

4. Zheng Y, Liu Y, Zhou Z (2013) From RSSI to CSI: indoor localization via channel response. Acm Comput Surv 46(2):1-32

5. Wei W, Liu AX, Shahzad M, Kang L, Lu S (2015) Understanding and modeling of wifi signal based human activity recognition. In: International conference on mobile computing and networking

6. Li M, Meng Y, Liu J, Zhu H, Liang H, Yao L, Na R (2016) When CSI meets public WiFi: Inferring your mobile phone password via WiFi signals. In: ACM Sigsac conference

7. Wang W, Liu AX, Shahzad M (2016) Gait recognition using WiFi signals. In: ACM international joint conference on pervasive and ubiquitous computing

8. Zeng Y, Pathak PH, Mohapatra P (2016) Wiwho: Wifi-based person identification in smart spaces. In: International conference on information processing in sensor networks

9. Ju W, Jiang H, Xiong J, Jamieson K, Xie B (2016) Lifs: Low human-effort, device-free localization with fine-grained subcarrier information. In: International conference on mobile computing networking

10. Daniel H. Linux 802.11n csi tool. http://dhalperi.github.io/linux80211n-csitool/installation.html

11. Halperin D, Hu W, Sheth A, Wetherall D (2011) Tool release: Gathering 802.11n traces with channel state information. ACM Sigcomm Comput Commun Rev 41(1):53-53

12. Xie Y. Atheros-CSI-tool. https://github.com/xieyaxiongfly/ Atheros-CSI-Tool

13. Sen S, Lee J, Kim KH, Congdon P (2013) Avoiding multipath to revive in building WiFi localization. In: Proceeding of the international conference on mobile systems

14. Wang G, Zou Y, Zhou Z, Wu K, Ni LM (2014) We can hear you with wi-fi. In: International conference on mobile computing and networking

15. Zhang Jie, Zheng Xiaolong, Tang Zhanyong, Xing TianZhang, Chen Xiaojiang, Fang Dingyi, Li Rong, Gong Xiaoqing, Chen Feng (2016) Privacy leakage in mobile sensing: Your unlock passwords can be leaked through wireless hotspot functionality. Mob Inf Syst 2016 (2016-4-11) 2016(12):1-14

16. Abdelnasser H, Youssef M, Harras KA (2015) Wigest: A ubiquitous wifi-based gesture recognition system. In: IEEE conference on computer communications

17. Platonov SS (2014) The telepathic phone: frictionless activity recognition from wifi-rssi. In IEEE international conference on pervasive computing communications

18. Ali K, Liu AX, Wang W, Shahzad M (2015) Keystroke recognition using wifi signals. In International conference on mobile computing and networking
19. Liu C, Lan Z, Liu Z, Liu K, Liu Y (2016) Lasagna: towards deep hierarchical understanding and searching over mobile sensing data. In: International conference on mobile computing networking

20. Yao S, Hu S, Zhao Y, Zhang A, Tarek A (2016) A unified deep learning framework for time-series mobile sensing data processing, Deepsense

21. Qian K, Wu C, Yang Z, Liu Y, He F (2018) Enabling contactless detection of moving humans with dynamic speeds using CSI. ACM Trans Embedded Comput Syst

22. Yang W, Wang X, Song A, Mao S (2018) Wi-wheat: Contact-free wheat moisture detection with commodity WiFi. In: 2018 IEEE international conference on communications (ICC 2018)

23. Praveen Kd, Tarachand A, Sekhar C (2019) Machine learning algorithms for wireless sensor networks: a survey. Inf Fusion 49:1-25

24. Cong S, Liu J, Liu H, Chen Y (2017) Smart user authentication through actuation of daily activities leveraging wifi-enabled iot. In: ACM international symposium on mobile Ad Hoc networking and computing

25. Liu H, Yan W, Jian L, Jie Y, Chen Y (2014) Practical user authentication leveraging channel state information (CSI). In: ACM symposium on information

26. Zhang J, Wei B, Hu W, Kanhere SS, Ariel T (2016) Human identification using wifi signal. In: IEEE international conference on pervasive computing and communication workshops

27. Cheng L, Wang J (2019) Walls have no ears: a non-intrusive wifibased user identification system for mobile devices. IEEE/ACM Trans Netw 27(1)

28. Jiang W, Dimitrios K, Wenyao X, Lu S, Xin M (2018) Towards environment independent device free human activity recognition. In: The 24th annual international conference on mobile computing and networking

29. Virmani A, Shahzad M (2017) Position and orientation agnostic gesture recognition using wifi. In: International conference on mobile systems

30. Tan S, Yang J (2016) Wifinger: leveraging commodity wifi for fine-grained finger gesture recognition. In: ACM international symposium on mobile ad hoc networking and computing

31. Venkatnarayan RH, Page G, Shahzad M (2018) Multi-user gesture recognition using wifi. In: The 16th ACM international conference on mobile systems, applications, and services

32. Wang W, Xu H, Alazab M, Gadekallu T, Han Z, Su C (2021) Blockchain-based reliable and efficient certificateless signature for iiot devices. In: IEEE transactions on industrial informatics

33. Wang T, Quan Y, Shen X, Gadekallu T, Wang W, Dev K (2021) A privacy-enhanced retrieval technology for the cloud-assisted internet of things. In: IEEE transactions on industrial informatics

34. Hamada N (2007) Image processing system for identifying car thieves

35. Chen D, Cho KT, Han S, Jin Z, Kang GS (2015) Invisible sensing of vehicle steering with smartphones. In: International conference on mobile systems

36. Yan W, Chen Y, Jie Y, Gruteser M, Martin R, Liu H, Liu L, Karatas C (2016) Determining driver phone use by exploiting smartphone integrated sensors. IEEE Trans Mob Comput 15(8):1965-1981

37. Wang Y, Yang J, Liu H, Chen Y, Gruteser M, Martin RP (2013) Sensing vehicle dynamics for determining driver phone use. In: Proceeding of the international conference on mobile systems

38. Zongjian H, Jiannong C, Xuefeng L, Shaojie T (2014) Who sits where? Infrastructure-free in-vehicle cooperative positioning via smartphones. Sensors 14(7):11605-11628

39. Raja M, Ghaderi V, Sigg S(2018) Wibot! in-vehicle behaviour and gesture recognition using wireless network edge. In: IEEE international conference on distributed computing systems

40. Peng H, Jia W (2018) Wifind: driver fatigue detection with fine-grained wi-fi signal features. In: Globecom IEEE global communications conference 
41. Raja M, Ghaderi V, Sigg S (2018) Detecting driver's distracted behaviour from wi-fi. In: 2018 IEEE 87th vehicular technology conference (VTC Spring)

42. Duan S, Yu T, Jie H (2018) Widriver: Driver activity recognition system based on WiFi CSI. Int J Wireless Inf Netw 25(3):1-11

43. Arshad S, Feng C, Elujide I, Zhou S, Liu Y (2018) Safedrive-fi: A multimodal and device free dangerous driving recognition system using Wifi. In: 2018 IEEE international conference on communications (ICC)

44. Wang F, Liu J, Gong W (2019) Wicar: wifi-based in-car activity recognition with multi-adversarial domain adaptation. In: The international symposium

45. Bai Y, Wang X (2020) Carin: Wireless CSI-based driver activity recognition under the interference of passengers. Proc ACM Interact Mob Wearable Ubiquitous Technol 4(1):1-28

46. Regani SD, Xu Q, Wang B, Wu M, Liu K (2019) Driver authentication for smart car using wireless sensing. IEEE Internet Things $\mathrm{J}$

47. Regani SD, Xu Q, Wang B, Wu M, Liu K (2019) In-car driver authentication using wireless sensing. In: ICASSP 2019-2019 IEEE international conference on acoustics, speech and signal processing (ICASSP)

48. Zheng T, Chen Z, Cai C, Luo J, Zhang X (2020) V2ifi: in-vehicle vital sign monitoring via compact RF sensing. Proc ACM Interactive Mob Wearable Ubiquitous Technol 4(2):1-27

49. Won M, Zhang S, Sang HS (2017) Witraffic: low-cost and nonintrusive traffic monitoring system using wifi. In: International conference on computer communication and networks
50. Wang J, Tong J, Gao Q, Wu Z, Sheng B, Wang H (2018) Devicefree vehicle speed estimation with wifi. IEEE Trans Veh Technol 99:1

51. Balamurugan NM, Senthilkumar M, Adimoolam M, Ayeelyan J, Gadekallu T, Wang W (2021) Doa tracking for seamless connectivity in beamformed iot-based drones. Comput Stand Interfaces 79

52. Liu J, Wang L, Fang J, Guo L, Lu B, Shu L. Multi-target intense human motion analysis and detection using channel state information. Sensors

53. Pei W, Guo B, Tong X, Zhu W, Yu Z (2017) Tinysense: Multi-user respiration detection using Wi-Fi CSI signals. In: IEEE international conference on E-health networking

Publisher's Note Springer Nature remains neutral with regard to jurisdictional claims in published maps and institutional affiliations. 